\title{
FGF1 stops diabetes in its tracks
}

Increasing evidence supports a key role of the brain in regulating glucose homeostasis, and as such the brain represents a promising potential target for the treatment of type 2 diabetes. Now, writing in Nature Medicine, Schwartz and colleagues report that central administration of a single dose of a fibroblast growth factor (FGF) family member, FGF1, induces sustained diabetes remission in rodents, without the risk of hypoglycaemia.

FGF1 has previously been implicated in glucose homeostasis, as mice lacking FGF1 that were challenged with a high-fat diet (HFD) developed insulin resistance. In addition, large systemic doses of FGF1 have been reported to effectively lower blood glucose for approximately 2 days.

Given that other FGF family members, specifically FGF19 and FGF21, transiently ameliorate hyperglycaemia in mice through effects on the brain, Schwartz and colleagues therefore set out to explore the potential antidiabetic efficacy of centrally administered FGF1.

The authors found that a single intracerebroventricular (i.c.v.) injection of recombinant FGF1, at a dose one-tenth of that needed for antidiabetic efficacy following peripheral injection, induced sustained diabetes remission in leptin-deficient $o b / o b$ mice. Blood glucose levels were normalized in the mice after 7 days and maintained for the rest of the 18 week study. This antidiabetic effect was not due to reduced food intake or weight loss, and importantly, there were no signs of hypoglycaemia.

The sustained antidiabetic effect of a single i.c.v. dose of FGF1 was reproduced in three additional groups of $o b / o b$ mice as well as in several other rodent diabetes models, including leptin-receptor-deficient $d b / d b$ mice, mice exposed to a combination of a HFD with a low dose of the pancreatic $\beta$-cell toxin streptozotocin and leptin-receptordeficient Zucker diabetic fatty rats.

Further analysis of the $o b / o b$ mouse model revealed that i.c.v. administration was acting to increase glucose clearance in the basal state into both the liver and skeletal muscle, without effects on basal hepatic glucose production or glucose tolerance. Intact insulin signalling appeared to be required for diabetes remission induced by the central action of FGF1.

Although the precise mechanisms mediating the antidiabetic effects of i.c.v. FGF1 remain unclear, the authors highlight a potential role for tanycytes (cells which line the third ventricle of the brain adjacently to the mediobasal hypothalamus), reporting their activation in FGF1-treated mice in conjunction with increased expression of the potent neuroprotective protein HSP25.

The authors note that they are unaware of any other non-surgical intervention capable of inducing sustained remission of type 2 diabetes. Importantly, this finding has translational potential as therapeutic FGF1 delivery to the central nervous system by the intranasal route has already been established in rodents.

Sarah Crunkhorn

ORIGINAL ARTICLE Scarlett J. M. et al. Central injection of fibroblast growth factor 1 induces sustained remission of diabetic hyperglycemia in rodents. Nat. Med. http://dx.doi.org/10.1038/ nm.4101 (2016) 